\title{
Review of: "Title-Effects of Catha Edulis Forsk on Spatial Cognition and correlation with serum electrolytes in Wild- type Male White Albino Rats"
}

Workineh Shibeshi Alemayehu

Potential competing interests: The author(s) declared that no potential competing interests exist.

\section{Review comments}

General comment:

The authors investigated the Effects of Catha Edulis Forsk on Spatial Cognition and correlation with serum electrolytes in Wild- type Male White Albino Rats. Controversy exists on true effect of khat on learning and memory/cognition. This study generated additional data that may help for generalization of knowledge in the field.

\section{Abstract}

- Write the title as 'Abstract'

- In methods : the terms subchronically (kesc) and subacutely (kesa) are vague, write the time in clear language

\section{Introduction}

- In-text citations lack uniformity for example, (8 and 9) and (5\& 11).

\section{Materials and methods:}

- What was the rationale for authors to use a mixture of diethyl ether and chloroform for extraction of Khat?

- Why Tween 80 was used to prepare khat juice? How this correlate with chewing of khat by people?

- Page 4, last paragraph, first line "The khat juice (khJ) was prepared from $12 \mathrm{~g} / \mathrm{kg}$ body weight (b.w) of fresh leaves" is not clear.

- Page 7, how was cardiac puncture done? were animals anesthetized?

\section{Results}

- Table 1: data presentation should be formatted. For example " $34.83^{* * *} \pm 2.46$ " is not correct. Can be corrected as $\mathbf{3 4 . 8 3} \mathbf{2} \mathbf{2 . 4 6 * * *}$. This correction should apply also for Tables 2,3,4.

- Table 1: statistical comparisons indicated that treatment doses compared to negative control. What about comparison between doses? Was there any dose-dependent effect?

- What was the role of ascorbic acid indicated in the legends of figures 2, and 4, which is neither described in the methodology?, which is not also found in figures themselves?

- Table 3: Tqt (s) is duplicated

\section{Discussion:}


- Why poor correlation was observed between average thigmotaxis and average escape latency? discuss 